\title{
Contribution of endoplasmic reticulum stress to the development and progression of diabetes mellitus
}

\begin{abstract}
Adaptation is the ability of the cell to sense disturbances, and initiates series of molecular reactions to survive. The main adaptations mechanisms involve atrophy, hypertrophy, hyperplasia and metaplasia. However, throughout these adaptation mechanisms, cells maintain their viability, nevertheless, under certain conditions; adaptive reactions may also produce organ damage. When the cell fails to adapt, it progresses to cell death programs (apoptosis, autophagy and necrosis) resulting in cellular dysfunction, parenchymal scarring and ultimately organ failure ensue. Disruption of these mechanisms from adaptation to cell death may cause unwanted inflammation and disease. ER stress has been contributed to pancreatic $\beta$-cell dysfunction and cell death resulting in the development of diabetes. ER stress has also been contributed to pancreatic $\beta$-cell dysfunction in more common forms of diabetes associated with obesity. ER stress also downregulates insulin hormone signaling pathways and contributes to the development of diabetes. Several studies have reported increased ER stress markers in pancreatic islets in rodent and in obese humans with type 2 diabetes mellitus. In these review we will discuss how ER stress (as a common early adaptation phase) and expression of unfolded proteins and their role in the development and progression of diabetes mellitus.
\end{abstract}

Keywords:cell stress, endoplasmic reticulum (ER) stress, extracellular vesicles (EVS), exosomes, horizontal gene transfer (HGT), unfolded protein response (UPR)
Volume 3 Issue 8 - 2016

\author{
Wael F Nassar \\ Department of Nephrology, Sahel Teaching Hospital, Egypt
}

Correspondence: Wael F Nassar, Department of Nephrology, Sahel Teaching Hospital, Cairo, Egypt,

Email Hegaz_wn@yahoo.com

Received: June 19, 2016 | Published: December 28, 2016

\section{Background}

The clinical presentation of any disease is the "functional expression and augmentation of disrupted cells". Cell stress can be infection, malnutrition, obesity, physical or mental exertion and other ecologic factors. The duration, severity and frequency of exposure to stressors are of particular importance in determining the progression of a disease from cell adaptation to cell death. ${ }^{1}$ The term cell injury is used to indicate a state in which the capacity for physiological adaptation is exceeded. This may occur when the stimulus is excessive or when the cell is no longer capable to adapt without suffering some form of damage. Cells are also subjected to different stresses relate to metabolic alterations. These conditions can lead to the accumulation of substances inside the cell (intracellular accumulation), such as fat (steatosis), proteins (e.g. unfolded) and more. The main cellular mechanisms of cell injury can be ATP depletion, loss of calcium homeostasis, oxidative stress (excess Reactive Oxygen Species) and damage to mitochondria with increased permeability of membranes and ER stress with excessive expression of UPR. ${ }^{2}$ The functions of the endoplasmic reticulum can be summarized as the synthesis and export of proteins and membrane lipids, but varies between cell type and cell function. The quantity of rough endoplasmic reticulum (RER) and sooth endoplasmic reticulum (SER) in a cell can slowly interchange from one type to the other, depending on the changing metabolic activities of the cell. ${ }^{3}$ ER stress has been contributed to pancreatic $\beta$-cell dysfunction and cell death resulting in the development of diabetes. ER stress has also been contributed to pancreatic $\beta$-cell dysfunction in more common forms of diabetes associated with obesity. Several studies have reported increased ER stress markers in pancreatic islets in rodent and in obese humans with type 2 diabetes mellitus. $^{4-6}$

Functions of the endoplasmic reticulum include folding of protein molecules and the transport of synthesized proteins to Golgi bodies. Only properly folded proteins are transported and secreted. Disturbances in redox regulation, calcium regulation, glucose deprivation, viral infection ${ }^{7}$ and proteins over-expression ${ }^{8}$ can lead to (ER stress), an adaptive state in which the folding of proteins slows, leading to intra-cytoplasmic accumulation of unfolded proteins. This stress is emerging as a potential cause of damage in hypoxia/ischemia, insulin resistance, and other disorders. The correct three-dimensional structure of translated proteins is essential to function, although some parts of functional proteins may remain unfolded. ${ }^{9}$ Failure to fold into native structure generally produces inactive proteins. However, some misfolded proteins have modified or toxic function. Several neurodegenerative disorders as Alzheimer's disease are believed to result from the accumulation of amyloid fibrils formed by Misfolded proteins. ${ }^{10}$ Many allergic conditions and autoimmune disorders are caused by incorrect folding of some proteins to which the immune system does not produce antibodies. ${ }^{11}$ In these review we will emphasize how ER stress and the expression of unfolded proteins may play a role in the development and progression of diabetes mellitus. Thus, understanding how pancreatic $\beta$-cells and insulin receptor cells respond to ER stress may help developing new strategies and open the gate for novel therapeutic modalities of the disease.

\section{Endoplasmic reticulum stress of the pancreatic $\beta$ cells}

Unfolded and misfolded proteins accumulation in the endoplasmic reticulum (ER) of $\beta$ cells leads to the activation of the unfolded protein response (UPR). UPR will transiently slow protein translation and activation transcription of many genes that impair the secretory function of the ER of $\beta$ cells. ${ }^{1}$ Three ER stress response sensors have been recognized, PERK, IRE1 and ATF6. These ER-transmembrane proteins can sense the accumulation of misfolded proteins. UPR is usually associated with a wide variety of expression and relative 
abundance of various ER secretions such as chaperone proteins, including protein disulfide isomerase (PDI), ERp29, the Hsp70 family member, calnexin, calreticulin and the peptidylpropyl isomerase family to correct folding of newly made proteins. ${ }^{12}$ Highly specialized cells such as insulin-secreting pancreatic $\beta$-cells have a unique chaperone expression profile. ${ }^{13}$

Prolonged persistence of ER stress will initiate apoptosis through a number of pathways, including prolonged expression of pro-apoptotic transcription factors such as CHOP, JNK stress kinase activation, and the IRE1- dependent degradation (or RIDD) activity of IRE1 that non-selectively degrades mRNAs of the ER membrane. ${ }^{14,15}$ ER stress has been contributed to pancreatic $\beta$-cell dysfunction, cell death and the development of diabetes. This is evident in rodents and humans that certain mutations in the insulin gene may cause misfolding of proinsulin in the ER. ${ }^{16-18}$ ER stress has also been contributed to pancreatic $\beta$-cell dysfunction in more common forms of T2D associated with obesity. Furthermore, enhanced chaperone capacity in pancreatic $\beta$-cells can improve $\beta$-cell function and protect $\mathrm{C} 57 \mathrm{Bl} / 6$ knockout mice from developing glucose intolerance in response to a high fat diet. ${ }^{19}$

\section{Endoplasmic reticulum stress at the level of insulin receptors}

ER stress significantly down regulate insulin receptor substrate- 1 (IRS-1), the substrate for insulin tyrosine kinase, which inhibit phosphorylation of tyrosine residues. ER stress stimulates phosphorylation and activates high levels of IRE-1 $\alpha$, which itself is phosphorylated and activated in the presence of ER stress. Furthermore, C-Jun N-terminal kinase (JNK) is also activated which subsequently phosphorylates serine residues of IRS-1, and thus inhibits insulin receptor signaling. This kinase cascade that is dependent on IRE-1 $\alpha$ and JNK mediates ER stress-induced inhibition of insulin action. ${ }^{20}$ Chronic inflammation associates obesity provides suitable stimuli for UPR pathway as a result of ER stress which down regulates insulin hormone signaling and hence, obese individuals become very likely to develop diabetes. ${ }^{21}$ Exercises can also impair endoplasmic reticulum homeostasis and express chaperones in response to exercise-induced ER stress. Muscular contraction during exercise release calcium from the sarcoplasmic reticulum (SR), a specialized ER vesicles which store and release calcium necessary for muscle contraction. Calcium released from SR is then interacts with calcineurin and calcium/ calmodulin-dependent kinases that activate transcription factors which regulate muscle contractions. PGC-1 alpha, a transcriptional coactivator, is expressed in muscles after acute and long-term exercises by co-activating ATF6 alpha. ${ }^{22}$ This transcription factor is important to increase the number and function of mitochondria necessary to slow oxidative stress of muscle fibers, as these are fatigue-resistant. Therefore, this UPR pathway mediates changes in muscles that have undergone endurance exercises by making them more resistant to fatigue and protecting them from future stress. ${ }^{23}$

\section{Conclusion}

In genetically predisposed patients, accumulation of environmental factors (malnutrition, obesity, physical or mental exertion and viral infections) and other ecologic factors induce a state of subclinical chronic inflammation. Cells constantly adapt to cope physiological demands and to maintain a homeostatic steady state. Disruption of these adaptation mechanisms superimposed to unwanted ER stress. ER stress has been contributed to pancreatic $\beta$-cell dysfunction and cell death resulting in the development of diabetes. ER stress also down regulates insulin hormone signaling pathways and contributes to the development of diabetes. Thus, understanding how pancreatic $\beta$-cells and insulin receptors cells respond to ER stress may help in developing new strategies to improve cell function and open a new gate for novel therapeutic modality of diabetes mellitus.

\section{Acknowledgements}

The author gratefully acknowledges Rashad S. Barsoum, Professor of Internal Medicine and Nephrology, Faculty of Medicine, Cairo University, Egypt, for his unlimited help and support. We wish also to acknowledge Dr. Mayar W N., Faculty of Medicine; October Six University, whose effort was behind most of the steps of this work.

\section{Conflict of interest}

The author of this review, declare that there is no conflict of interest that could be perceived as prejudicing the impartiality of the research. We fully declare that no financial or other potential conflict of interest.

\section{References}

1. Walter P, Ron D. The unfolded protein response: from stress pathway to homeostatic regulation. Science. 2011;334:1081-1086.

2. Delepine $M$, Nicolino $M$, Barrett $T$, et al. EIF2AK3, encoding translation initiation factor 2-alpha kinase 3, is mutated in patients with Wolcott-Rallison syndrome. Nat Genet. 2000;25(4):406-409.

3. Harding HP, Zeng H, Zhang Y, et al. Diabetes Mellitus and exocrine pancreatic dysfunction in Perk $-/-$ mice reveals a role for translational control in secretory cell survival. Mol Cell. 2001;7:1153-1163.

4. Huang CJ, Haataja L, Gurlo T, et al. Induction of endoplasmic reticulum stress-induced beta-cell apoptosis and accumulation of polyubiquitinated proteins by human islet amyloid polypeptide. Am J Physiol Endocrinol Metab. 2007;293(6):E1656-E1662.

5. Laybutt DR, Preston AM, Akerfeldt MC, et al. Endoplasmic reticulum stress contributes to beta cell apoptosis in type 2 diabetes. Diabetologia. 2007;50(4):752-763.

6. Sachdeva MM, Claiborn KC, Khoo C, et al. Pdx1 (MODY4) regulates pancreatic beta cell susceptibility to ER stress. PNAS 2009;106(45):19090-19095.

7. Xu C, Bailly-Maitre B, Reed JC. Endoplasmic reticulum stress: cell life and death decision. J Clin Invest. 2005;115(10):2656-2664.

8. Kober L, Zehe C, Bode J. Development of a novel ER stress based selection system for the isolation of highly productive clones. Biotechnol Bioeng. 2012;109(10):2599-2611.

9. Jeremy M Berg, John L Tymoczko, Lubert stryer. Web content by Neil D. Clarke. "3. Protein structure and function". Biochemistry. San Francisco, USA: WH Freeman; 2002.

10. Dennis J Selkoe. Folding proteins in fatal ways. Nature. 2003;426(6968):900-904.

11. Alberts, Bruce, Dennis Bray, et al. Protein Structure and Function. Essential Cell Biology. 3rd ed. New York, USA: Garland Science, Taylor and Francis Group, LLC; 2012. p. 120-170.

12. Yusta B, Baggio LL, Estall JL, et al. GLP-1 receptor activation improves beta cell function and survival following induction of endoplasmic reticulum stress. Cell Metab. 2006;4(5):391-406.

13. Powers ET, Morimoto RI, Dillin A, et al. Biological and chemical approaches to diseases of proteostasis deficiency. Annu Rev Biochem. 2009;78:959-991. 
14. Oslowski CM, Hara T, O'Sullivan-Murphy B, et al Thioredoxininteracting protein mediates ER stress-induced beta-cell death through initiation of the inflammasome. Cell Metab. 2012;16(2):265-273.

15. Lerner AG, Upton JP, Praveen PV, et al. IRE1alpha induces thioredoxininteracting protein to activate the NLRP3 inflammasome and promote programmed cell death under irremediable ER stress. Cell Metab. 2012;16(2):250-264

16. Han D, Lerner AG, Vande Walle L, et al. IRE1alpha kinase activation modes control alternate endoribonuclease outputs to determine divergent cell fates. Cell. 2009;138(3):562-575.

17. Zinszner H, Kuroda M, Wang $\mathrm{X}$, et al. CHOP is implicated in programmed cell death in response to impaired function of the endoplasmic reticulum. Genes Dev. 1998;12(7):982-995.

18. Urano F, Wang X, Bertolotti A, et al. Coupling of stress in the ER to activation of JNK protein kinases by transmembrane protein kinase IRE1. Science. 2000;287(5453):664-666.
19. Tabas I, Ron D. Integrating the mechanisms of apoptosis induced by endoplasmic reticulum stress. Nat Cell Biol. 2011;13(3):184-190.

20. Lee AH, Iwakoshi NN, Anderson $\mathrm{KC}$, et al. Proteasome inhibitors disrupt the unfolded protein response in myeloma cells. Proc Natl Acad Sci USA. 2003;100(17):9946-9951.

21. Teodoro-Morrison T, Schuiki I, Zhang L, et al. GRP78 overproduction in pancreatic beta cells protects against high fat diet-induced diabetes in mice. Diabetologia. 2013;56(5):1057-1067.

22. Hartley T, Siva M, Lai E, et al. Endoplasmic reticulum stress response in an INS-1 pancreatic beta-cell line with inducible expression of a folding-deficient proinsulin. BMC Cell Biol. 2010;11:59.

23. Lee AS. Coordinated regulation of a set of genes by glucose and calcium ionophores in mammalian cells. Trends Biochem Sci. 1987;12:20-23. 